\title{
Effect of Roller Geometry on Roller Bearing Load-Life Relation
}

\author{
Fred B. Oswald \\ NASA Glenn Research Center \\ Erwin V. Zaretsky \\ NASA Glenn Research Center
}

Joseph V. Poplawski

Poplawski \& Associates

STLE 67th Annual Meeting \& Exhibition

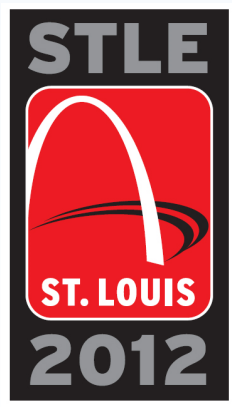




\section{Life Modeling of Roller Bearings}

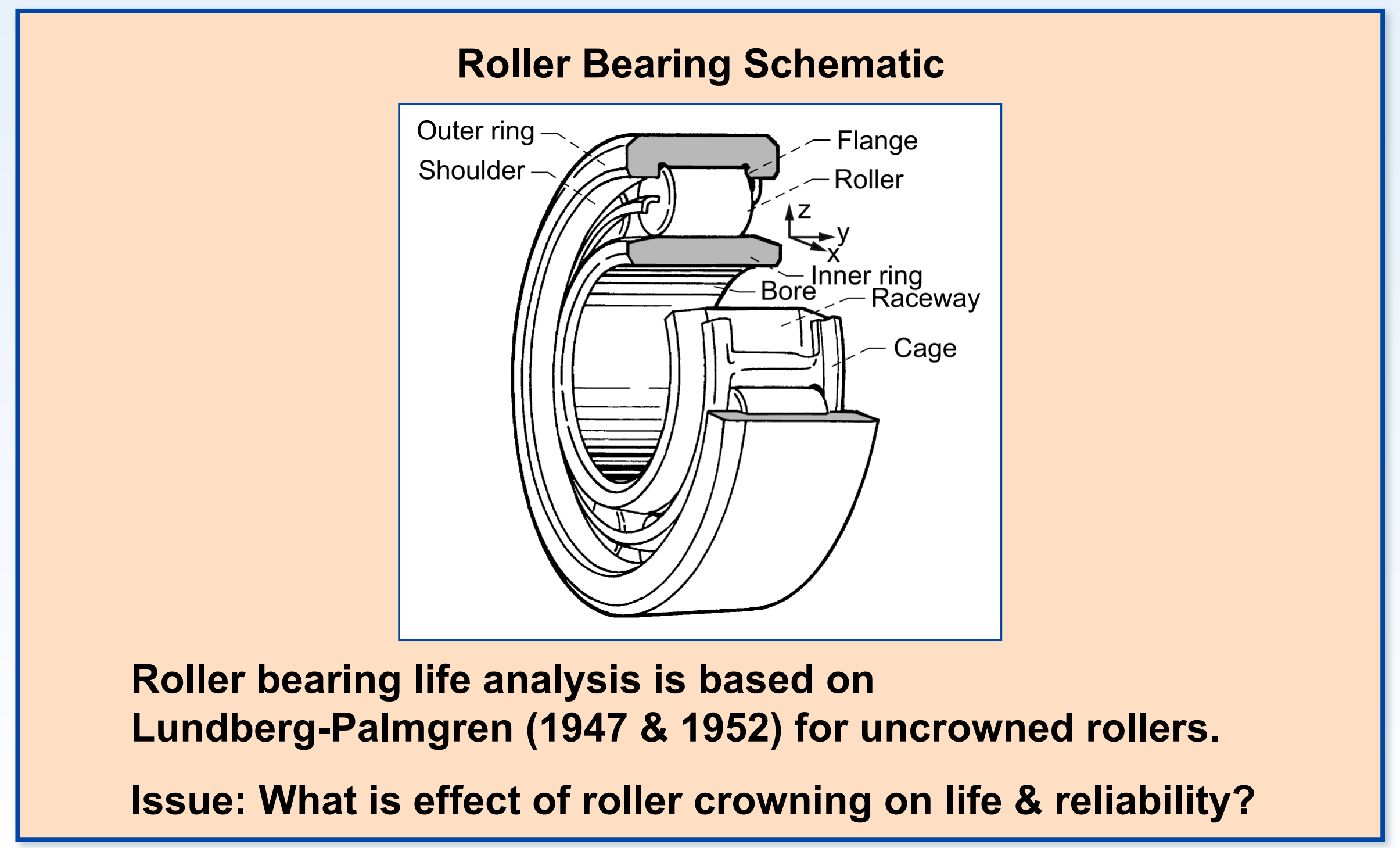




\section{Life Modeling of Roller Bearings}

Lundberg-Palmgren (1947) rolling bearing life relation:

$$
L \sim\left(\frac{1}{\tau}\right)^{c / m}\left(\frac{1}{V}\right)^{1 / m}(z)^{h / m} \sim\left(\frac{1}{S_{\max }}\right)^{n} \sim\left(\frac{1}{P}\right)^{p}
$$

where:

$L=$ Life

$\tau=$ Critical shear stress

$S_{\text {max }}=$ Max. Hertz stress

$c$ = shear stress-life exponent

$n=$ Exponent

$m=$ Weibull slope

$P \quad=$ Radial load

exponent

$V=$ Stressed volume

$z=$ Depth to crit. shear stress

$h=$ Exponent

with LP model, $n=8$ and $p=4$ for line contact 


\section{Life Modeling of Roller Bearings}

Lundberg \& Palmgren (1947) radial bearing load-life relation:

where:

$$
L=\left(\frac{C}{P}\right)^{p}
$$

$L=$ Life

$C=$ Dynamic load capacity

$\boldsymbol{P}=$ Applied radial load

exponent

$p=\mathbf{3}$ for either ball bearings or roller bearings

This relation was semi-empirical - based on life tests 


\section{Life Modeling of Roller Bearings}

Lundberg \& Palmgren (1952) revised cylindrical roller bearing load-life relation:

$$
L=\left(\frac{C}{P}\right)^{p}
$$

$p=3$ for pure point contact with both rings

$p=\mathbf{4}$ for pure line contact with both rings

$p=10 / 3=3.33$ for mixed point and line contact

ANSI/ABMA and ISO Standards use $p=\mathbf{3 . 3 3}$ for roller bearings 


\section{Life Modeling of Roller Bearings}

Zaretsky (1996) modified the LP life equation, eliminating [1/z $]^{\text {h }}$

where:

$$
L \sim\left(\frac{1}{\tau}\right)^{c}\left(\frac{1}{V}\right)^{1 / m} \sim\left(\frac{1}{S_{\max }}\right)^{n} \sim\left(\frac{1}{P}\right)^{p}
$$

$L=$ Life

$V \quad=$ Stressed volume

$\tau=$ Critical shear stress

$S_{\text {max }}=$ Max. Hertz stress

$C=$ shear stress-life exponent

$n$ = Exponent

$m=$ Weibull slope

$p \quad=$ Load-life exponent

with Zaretsky model, $n=10$ and $p=5$ for line contact

Zaretsky model better fits post-1960, vacuum-processed steels 


\section{Objectives}

- Investigate effect of roller profiles on load/life and stress/life relation for cylindrical roller bearings

- Flat (uncrowned)

- Aerospace crown

- Chamfered

- Full crown, $r=150 D$

- Full crown, $r=100 D$

Results based on 210-size cylindrical roller bearing: Bore $50 \mathrm{~mm}$, OD $90 \mathrm{~mm}$, width $20 \mathrm{~mm}$, roller dia. \& length $=13 \mathrm{~mm}$ 


\section{Life Modeling of Roller Bearings}

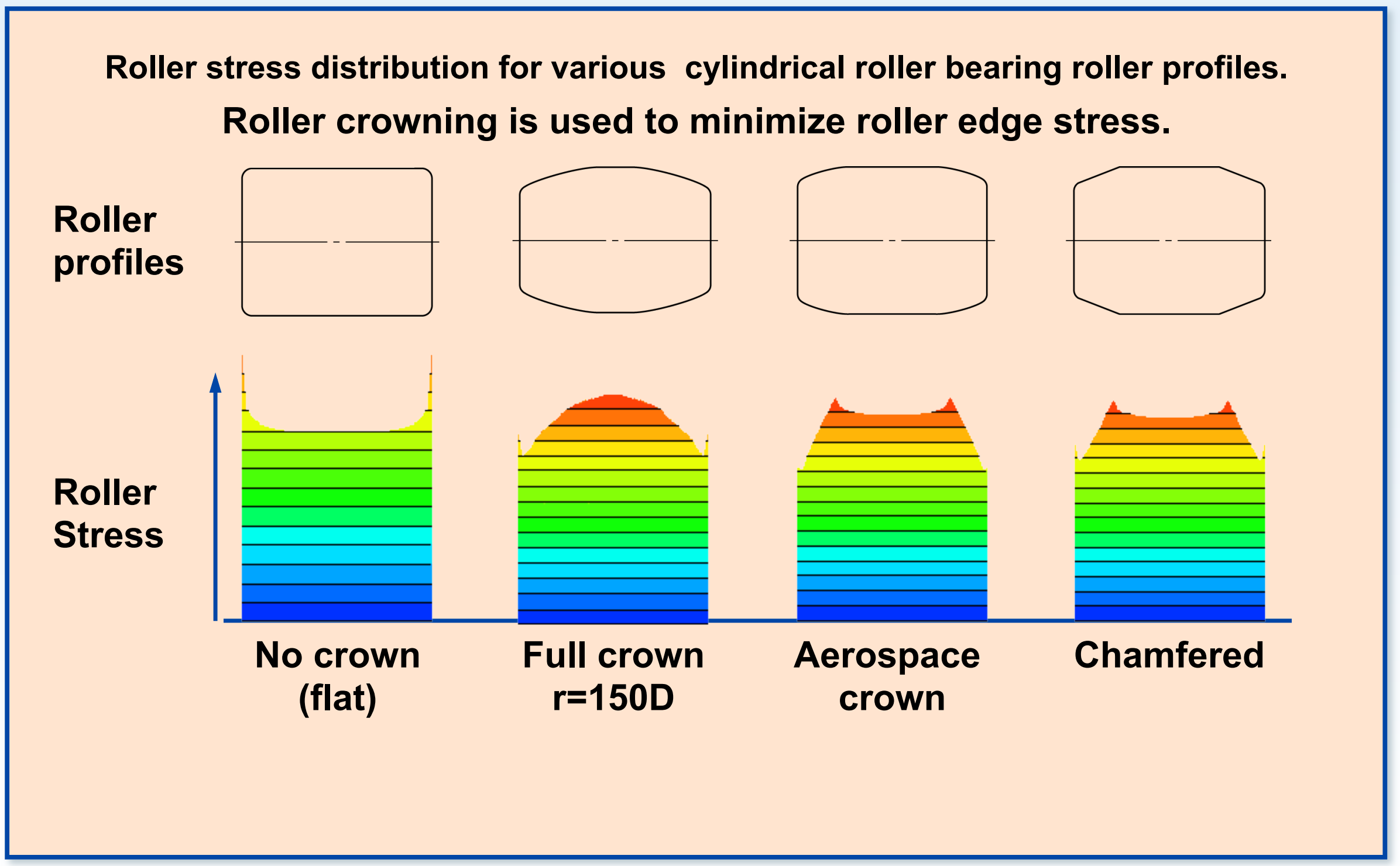




\section{Load-Life Relation - Lundberg-Palmgren Model}

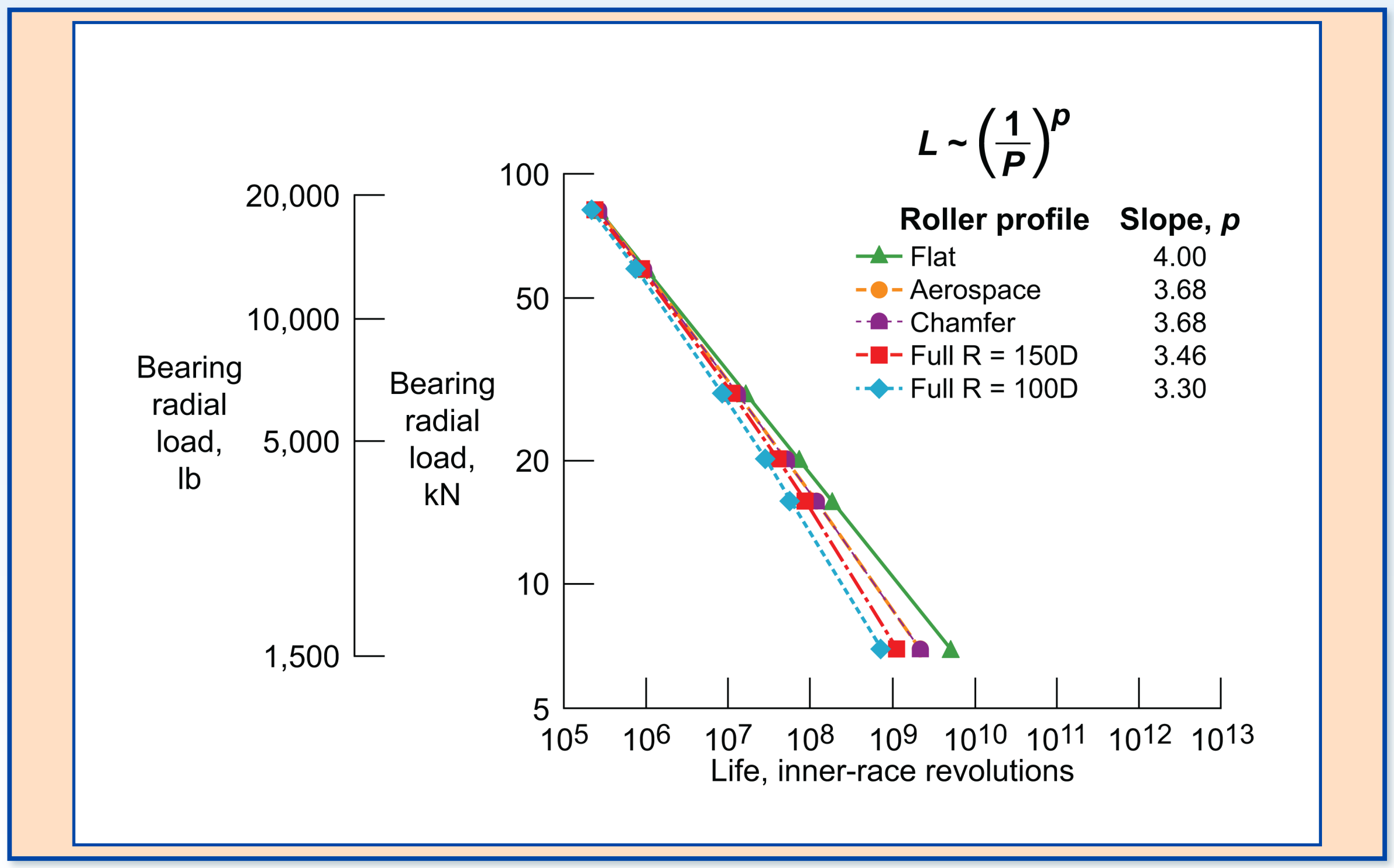




\section{Hertz Stress-Life Relation - Lundberg-Palmgren Model}

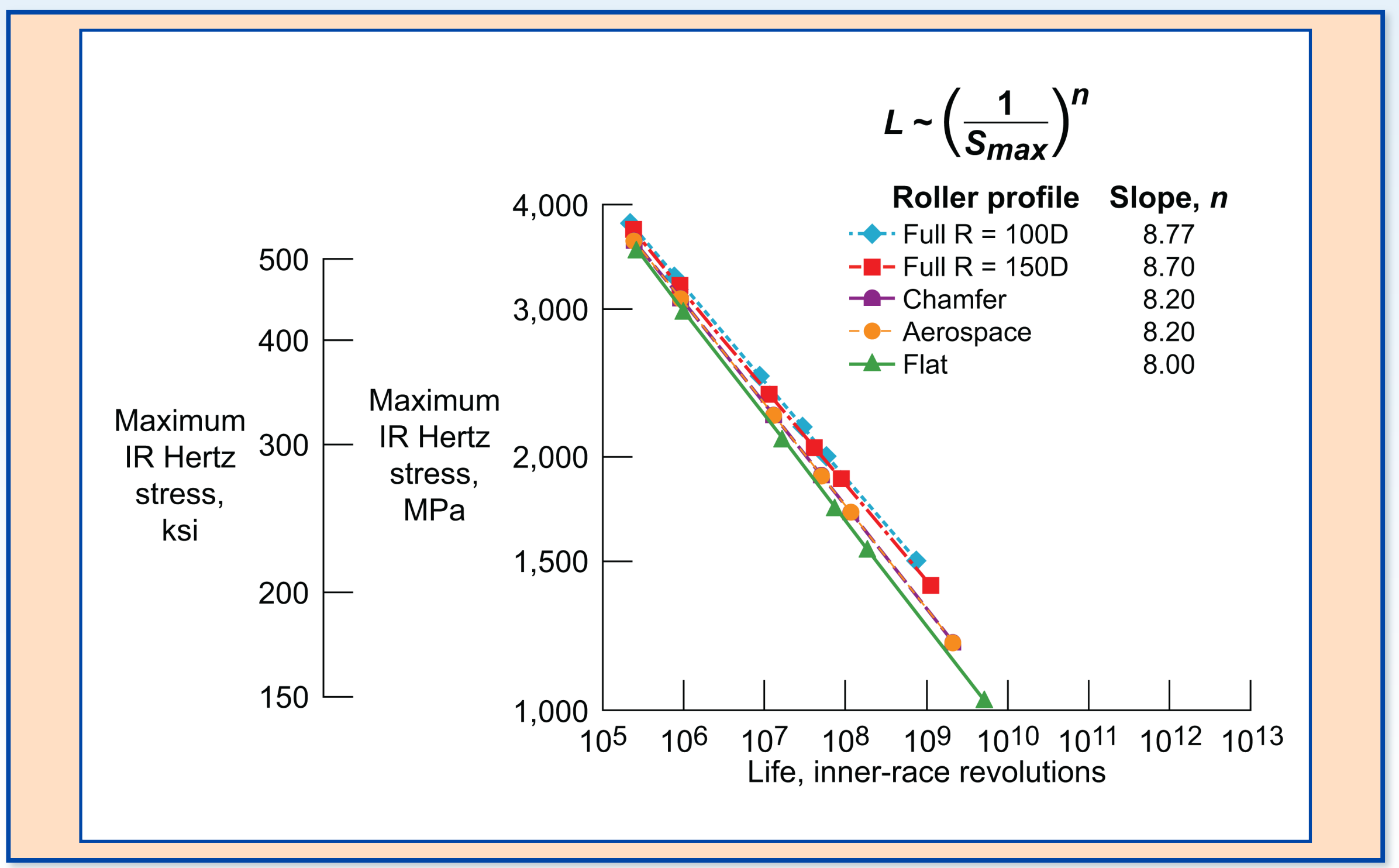




\section{Load-Life Relation - Zaretsky Model}

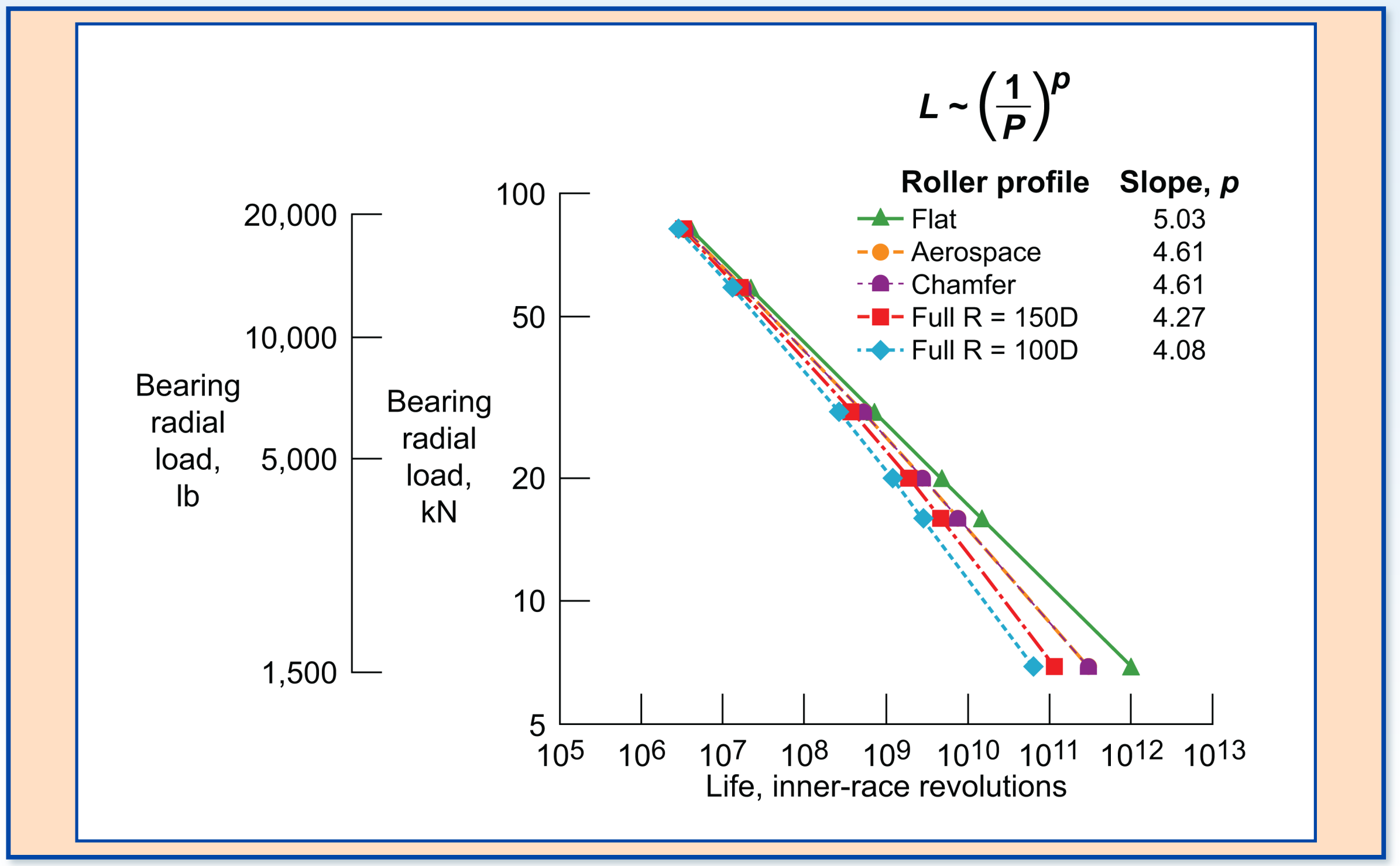




\section{Hertz Stress-Life Relation - Zaretsky Model}

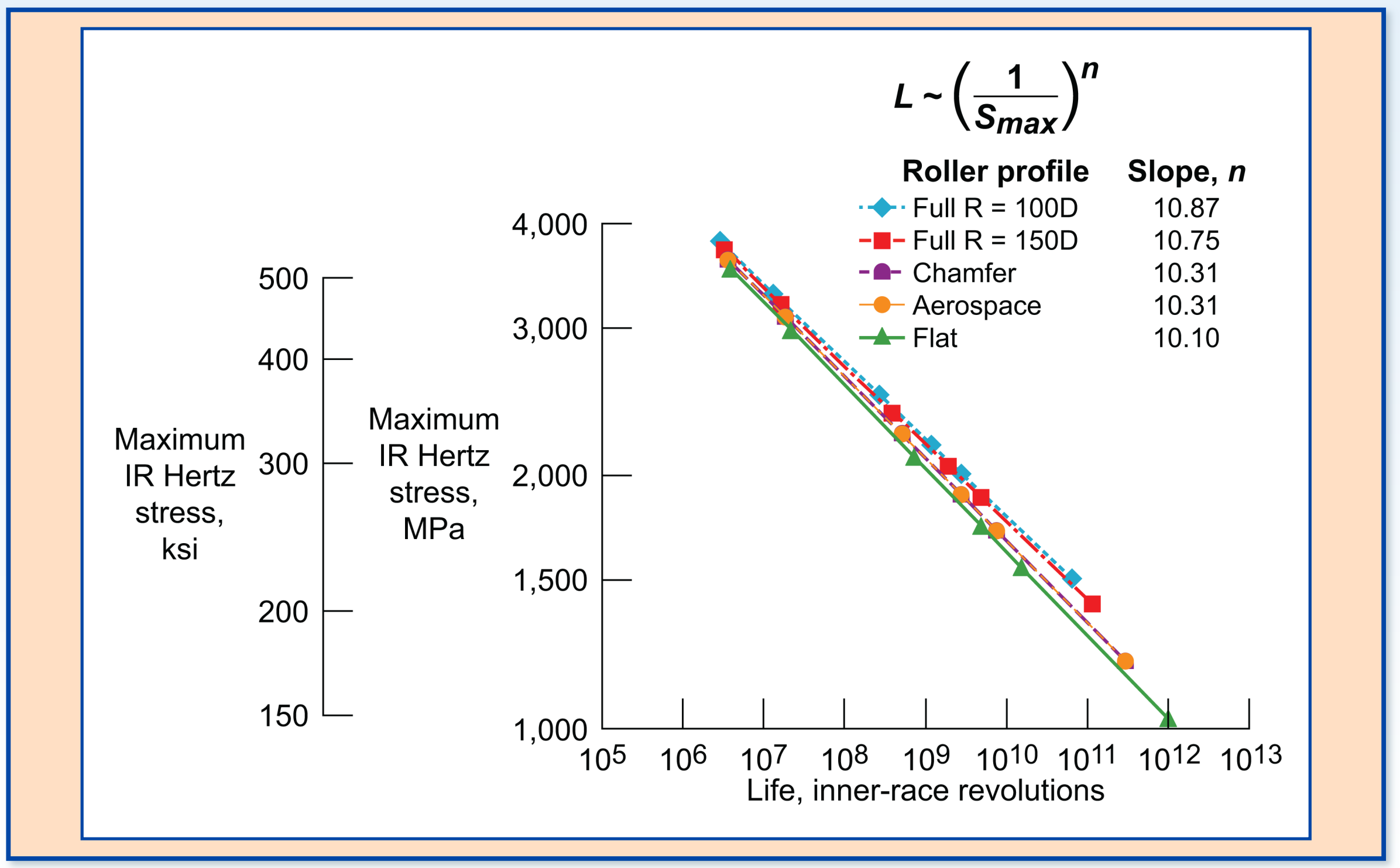




\section{Comparison of Lundberg-Palmgren and Zaretsky Models}

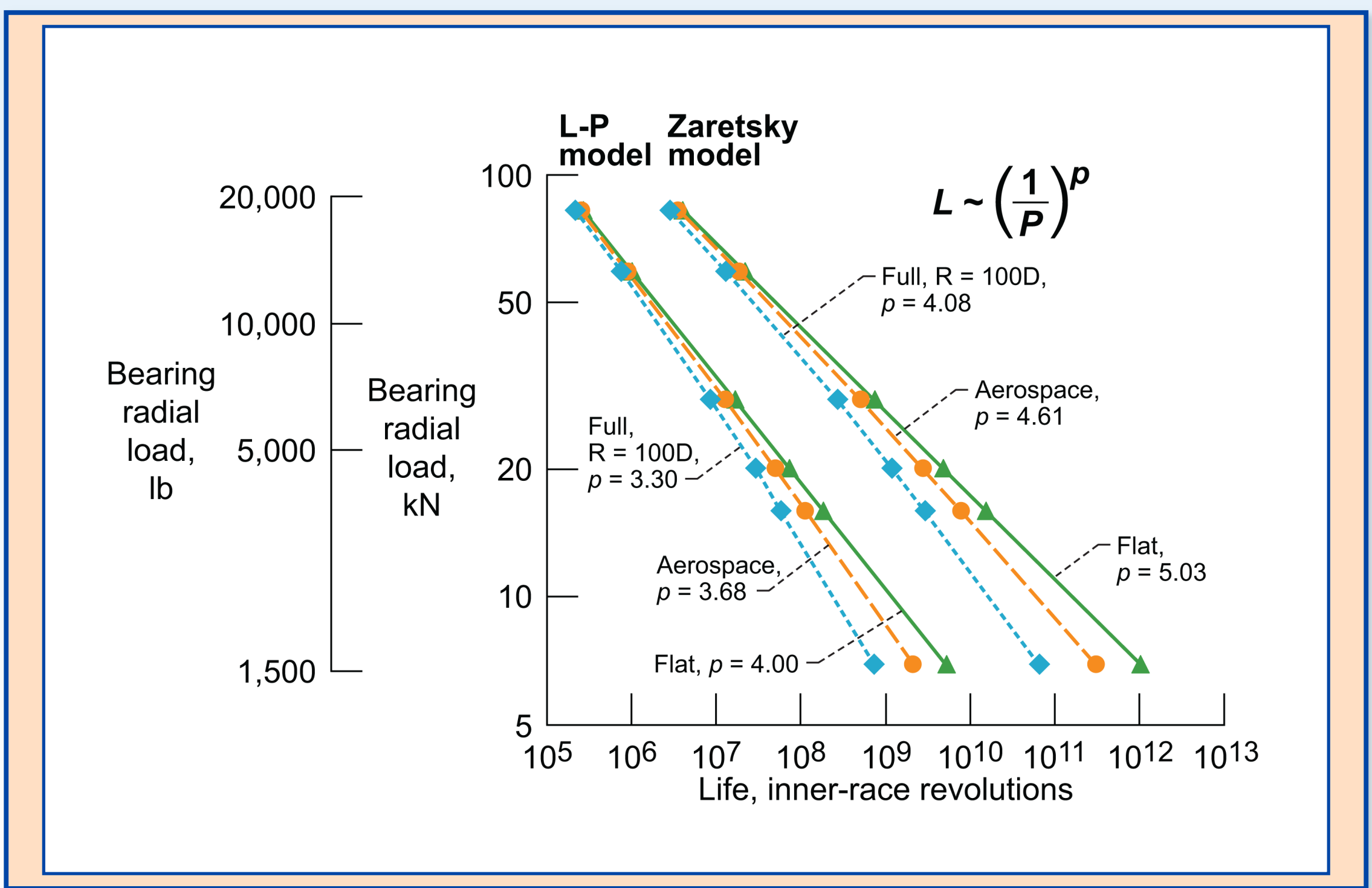




\section{Comparison of Lundberg-Palmgren and Zaretsky Models}

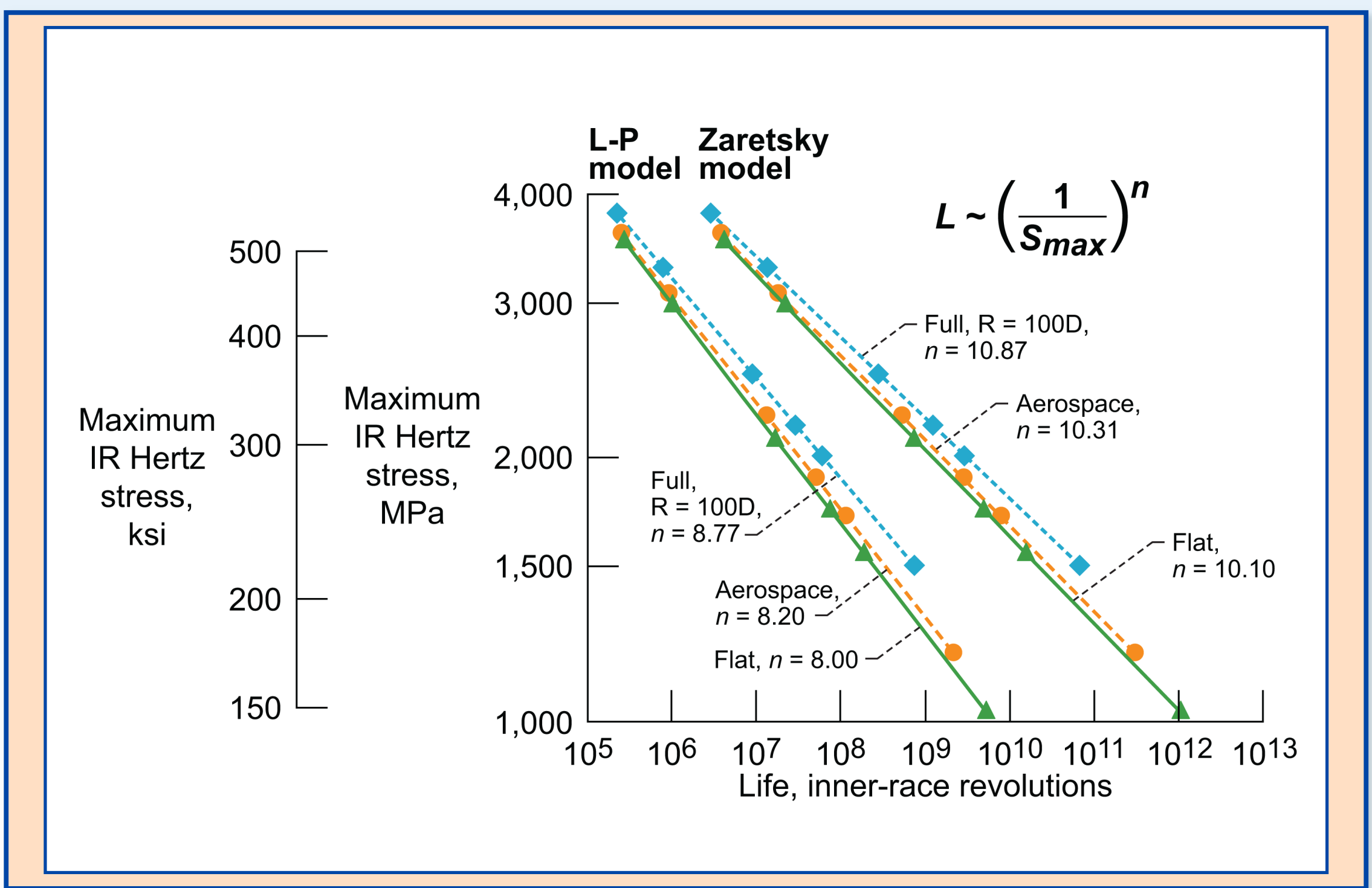




\section{Comparison of Lundberg-Palmgren and Zaretsky Models}

Relative Life $=1.0$ for $\mathrm{C} / \mathrm{P}=4.7$ for flat roller

where $S_{\max }=1556 \mathrm{MPa}(226 \mathrm{ksi})$

\begin{tabular}{|l|c|c|c|c|c|c|}
\hline \multirow{2}{*}{ Roller Profile } & \multicolumn{3}{|c|}{ Lundberg-Palmgren } & \multicolumn{3}{c|}{ Zaretsky } \\
\cline { 2 - 7 } & $p$ & $n$ & Rel. Life & $p$ & $n$ & Rel. Life \\
\hline Flat & 4.00 & 8.00 & 1.0 & 5.03 & 10.10 & 83 \\
\hline Aero. \& Chamfer & 3.68 & 8.20 & 0.6 & 4.61 & 10.31 & 43 \\
\hline Full, R=150D & 3.46 & 8.70 & 0.5 & 4.27 & 10.75 & 27 \\
\hline Full, R=100D & 3.30 & 8.77 & 0.3 & 4.08 & 10.87 & 16 \\
\hline Full, R=50D & 3.10 & 8.77 & 0.2 & 3.82 & 10.84 & 6 \\
\hline
\end{tabular}

$R=$ crown radius of curvature

$\mathrm{D}=$ roller diameter 


\section{Summary of Results}

- For Flat rollers, Zaretsky load-life exponent $p=5$ compared to $p=4$ for LP model

- Confirmed LP model for Full Crown $p=3.33$ Zaretsky model for Full Crown $p=4.3$

- Aerospace or Chamfered Crown $p=3.7$ for LP model and $p=\mathbf{4 . 6}$ for Zaretsky model

- Zaretsky model predicts life 83 times higher than LP model for flat rollers at moderate load 


\section{Published Hertz Stress-Life Data}

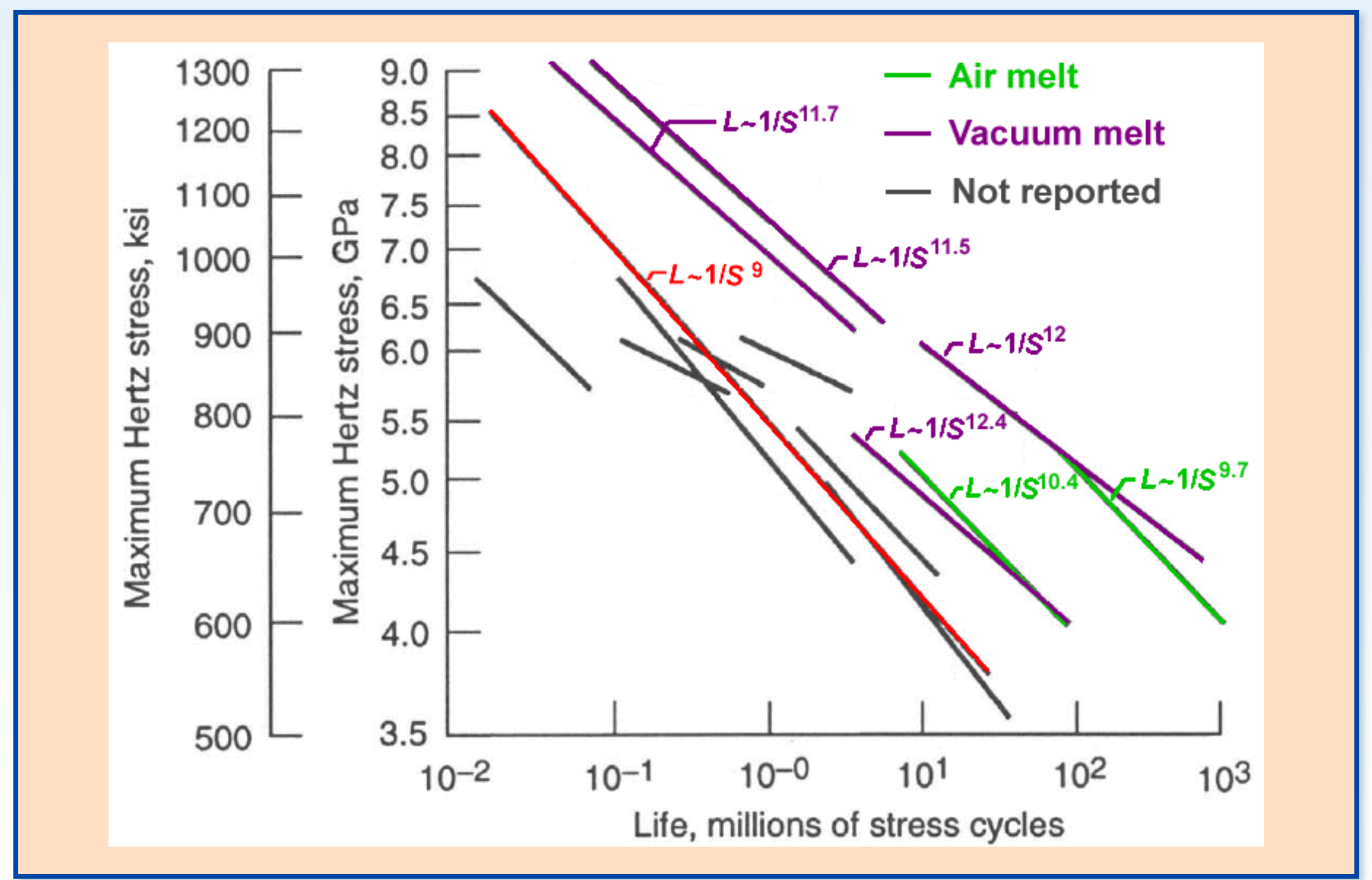

